\title{
Nanomechanical investigation of thin-film electroceramic/metal-organic framework multilayers
}

\author{
James P. Best, ${ }^{1, a)}$ Johann Michler, ${ }^{1}$ Jianxi Liu, ${ }^{2}$ Zhengbang Wang, ${ }^{2}$ Manuel Tsotsalas, ${ }^{2}$ \\ Xavier Maeder, ${ }^{1}$ Silvana Röse, ${ }^{3,4}$ Vanessa Oberst, ${ }^{5}$ Jinxuan Liu, ${ }^{2}$ Stefan Walheim, ${ }^{6}$ \\ Hartmut Gliemann, ${ }^{2}$ Peter G. Weidler, ${ }^{2}$ Engelbert Redel, ${ }^{2, a)}$ and Christof Wöll ${ }^{2, a)}$ \\ ${ }^{1}$ Empa, Swiss Federal Laboratories for Materials Science and Technology, Laboratory for Mechanics of \\ Materials and Nanostructures, Feuerwerkerstrasse 39, CH-3602 Thun, Switzerland \\ ${ }^{2}$ Institute of Functional Interfaces (IFG), Karlsruhe Institute of Technology (KIT), Hermann-von-Helmholtz- \\ Platz 1, 76344 Eggenstein-Leopoldshafen, Germany \\ ${ }^{3}$ Preparative Macromolecular Chemistry, Institute for Chemical Technology and Polymer Chemistry (ICTP), \\ Karlsruhe Institute of Technology (KIT), Engesserstrasse 18, 76128 Karlsruhe, Germany \\ ${ }^{4}$ Institute for Biological Interfaces (IBG), Karlsruhe Institute of Technology (KIT), Herrmann-von-Helmholtz- \\ Platz 1, 76344 Eggenstein-Leopoldshafen, Germany \\ ${ }^{5}$ Institute of Applied Materials (IAM), Karlsruhe Institute of Technology (KIT), Hermann-von-Helmholtz-Platz 1, \\ 76344 Eggenstein-Leopoldshafen, Germany \\ ${ }^{6}$ Institute of Nanotechnology (INT), Karlsruhe Institute of Technology (KIT), Hermann-von-Helmholtz-Platz 1, \\ 76344 Eggenstein-Leopoldshafen, Germany
}

(Received 21 April 2015; accepted 12 August 2015; published online 8 September 2015)

\begin{abstract}
Thin-film multilayer stacks of mechanically hard magnetron sputtered indium tin oxide (ITO) and mechanically soft highly porous surface anchored metal-organic framework (SURMOF) HKUST-1 were studied using nanoindentation. Crystalline, continuous, and monolithic surface anchored MOF thin films were fabricated using a liquid-phase epitaxial growth method. Control over respective fabrication processes allowed for tuning of the thickness of the thin film systems with a high degree of precision. It was found that the mechanical indentation of such thin films is significantly affected by the substrate properties; however, elastic parameters were able to be decoupled for constituent thin-film materials $\left(E_{I T O} \approx 96.7 \mathrm{GPa}, E_{H K U S T-1} \approx 22.0 \mathrm{GPa}\right)$. For indentation of multilayer stacks, it was found that as the layer thicknesses were increased, while holding the relative thickness of ITO and HKUST-1 constant, the resistance to deformation was significantly altered. Such an observation is likely due to small, albeit significant, changes in film texture, interfacial roughness, size effects, and controlling deformation mechanism as a result of increasing material deposition during processing. Such effects may have consequences regarding the rational mechanical design and utilization of MOF-based hybrid thin-film devices. (C) 2015 AIP Publishing LLC.

[http://dx.doi.org/10.1063/1.4930141]
\end{abstract}

Monolithic surface anchored metal-organic framework (SURMOF) thin films, constructed from inorganic metal (or metal/oxo) nodes periodically spaced by organic linkers, ${ }^{1-4}$ are a particularly interesting class of novel material for the fabrication of new application driven devices. ${ }^{5}$ MOFs are also referred to as porous coordination polymers (PCPs) ${ }^{6}$ and were originally developed to provide highly porous and accessible network materials with large gas storage and separation capacities for light gases (e.g., $\mathrm{H}_{2}$ and $\mathrm{CH}_{4}$ ). ${ }^{7}$ Additional applications have more recently been found in chemical sensing, drug delivery, artificial light harvesting, ${ }^{8}$ and carbon capture. $^{9-11}$ Monolithic SURMOF coatings have been shown to be stable to temperatures typically exceeding $200^{\circ} \mathrm{C}$ (exceeding $500{ }^{\circ} \mathrm{C}$ for ZIF-8 based SURMOFs ${ }^{12}$ ), and when combined with their dielectric, ${ }^{13}$ optical, ${ }^{14}$ and mechanical ${ }^{3}$ properties, MOF coatings offer a promising basis for future applications and devices. For practical realization of MOFbased devices, the deposition of well-defined films on substrates is required. ${ }^{5,15}$ Scalability and long term stability of

\footnotetext{
a) Authors to whom correspondence should be addressed. Electronic addresses: james.best@empa.ch; engelbert.redel@kit.edu; and christof.woell@kit.edu
}

assembled devices, as well as integration during fabrication processes, will however strongly depend on the material mechanical properties of such films. Whereas a few reports have been published on MOF mechanical properties, ${ }^{16}$ the number of reports on the mechanical properties of MOF thin films is considerably less. ${ }^{16-19}$

Hybrid materials systems are inherently multifunctional. Here, we focus on combining soft metalorganic thin films and hard inorganic metal oxide materials. The mechanical properties of such multilayers are of considerable interest, since multilayer formation from hard and soft materials may allow for an improvement in the material toughness. ${ }^{20}$ Here, we focus on MOF/indium tin oxide (ITO) multilayers which have been recently used to realize an optical sensor (Figure 1). ${ }^{21}$ The multilayers comprised HKUST1 (or $\mathrm{Cu}_{3}(1,3,5 \text {-benzenetricarboxylic acid) })_{2}$ ) SURMOF thinfilms (Figure 1(a)) layered consecutively with electrically conductive ITO (Figure 1(b)). The fabrication of the monolithic, hybrid SURMOF-based multi-layered architectures begins with HKUST-1 SURMOF growth with thicknesses ranging from 80 to $135 \mathrm{~nm}$ on a modified Si-substrate using a liquid phase epitaxy (LPE) technique (for experimental details, see the supplementary material ${ }^{35}$ ). An ITO layer 


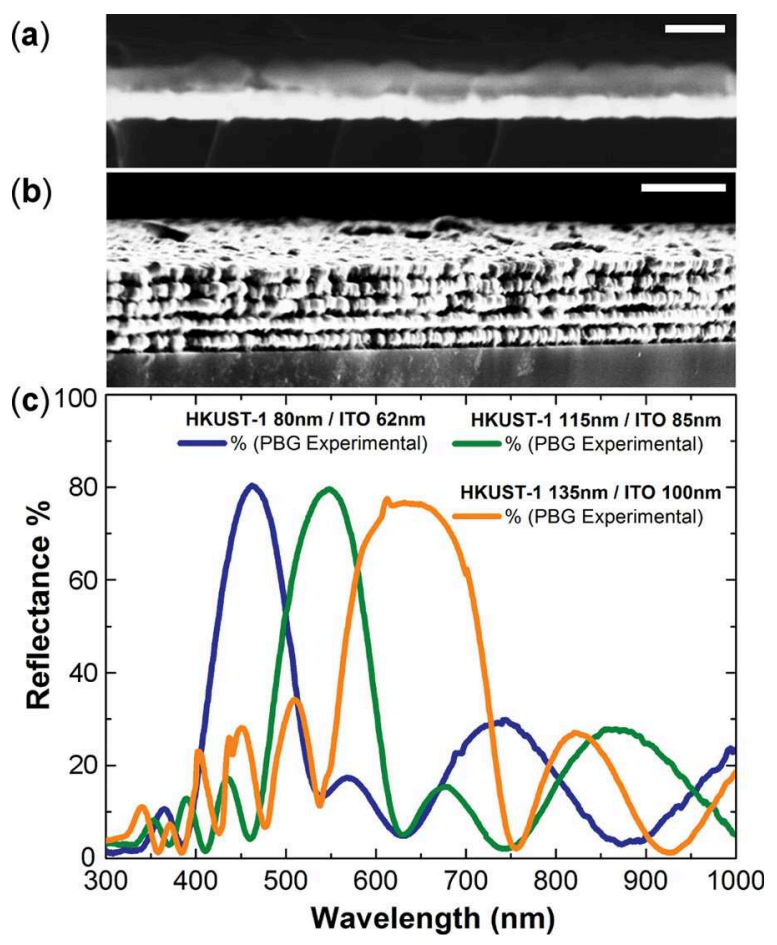

FIG. 1. SEM cross-sections of a monolithic grown single layered HKUST-1 thin-film (a) (scale bar $200 \mathrm{~nm}$ ) and a 5 bilayer (corresponding to the yellow track in (c)) HKUST-1/ITO hybrid architecture (b) (scale bar $2 \mu \mathrm{m}$ ). Photonic band gap (PGB) properties of stacked HKUST-1 and ITO thin films (c). Adapted with permission from Liu et al., Chem. Mater. 27, 1991-1996 (2015). Copyright 2015 American Chemical Society.

with a thickness of 62 to $100 \mathrm{~nm}$ is then sputter-deposited using r.f. magnetron sputtering. Due to free $\mathrm{OH}$-groups on the surface of deposited ITO layers, the growth of subsequent HKUST-1 SURMOF layers can then be achieved using LPE, and the process is repeated until the desired number of layers is obtained.

Multilayered hybrid systems comprising "soft" HKUST1 layers and "hard" ITO layers can be precisely controlled regarding thickness, by adjusting the number of deposition LPE-cycles, or optimizing the sputtering time, respectively. In addition to optical effects (Figure 1(c)), soft/hard interfacial and morphological effects can therefore be magnified via tuning of the individual layer thicknesses. The compact, monolithic, and high quality nature of the 1D multilayered hybrid architecture also allows us to exploit the favorable mechanical properties of HKUST-1 SURMOF materials in regard to their elastic properties. ${ }^{17}$ Herein, we first investigate the mechanical parameters of single-component ultrathin monolithic HKUST-1 SURMOFs and ITO films, as well as multilayered hybrid systems whereby exploring the influence of interfacial morphology on the mechanical response. There currently exist no mechanical results reported on multilayer and/or hybrid material compositions incorporating SURMOFs, even though the mechanical properties are vital for the fabrication and utilization of any device.

To better understand the mechanical properties of the hybrid electroceramic/SURMOF systems, we first investigated the constituent single layer HKUST-1 and ITO thinfilms by nanoindentation with a sharp diamond Berkovich tip. Films with five different thicknesses were prepared for both HKUST-1 $(38,75,106,130$, and $193 \mathrm{~nm})$ and ITO (70, $122,255,473$, and $1013 \mathrm{~nm})$. The Hardness $(H)$ and Young's modulus $(E)$ was evaluated using the approach outlined by Oliver and Pharr, ${ }^{22}$ and investigated as a function of normalized thickness (contact depth by film thickness). Substrate effects are likely to cause significant error for both ITO and HKUST-1 films on silicon at contact depths above approximately $10 \%$ of the film thickness $\left(h_{c} / t>0.1\right)$. As there are negative influences of the tip apex and film roughness at low contact depths for such thin films, it is therefore important to take into account stress-field interaction with the substrate, and extrapolate the respective relative moduli to an ideal case where $h_{c} / t=0$. A modified form of the equation presented by Perriot and Barthel (Eq. (1)) was therefore employed, where $E_{f}^{*}$ is the indentation (or reduced) modulus of the film where $E^{*}=E /\left(1-\nu^{2}\right), E^{*}{ }_{s}$ is the indentation modulus of the substrate, $\nu$ is the Poisson's ratio, while $x_{0}$ and $n$ are variable curve fitting parameters. ${ }^{23}$ The contact depth $h_{c}$ was assumed to be proportional to the contact radius. Silicon substrate properties were taken from the literature, ${ }^{24}$

$$
E_{e q}^{*}=E_{f}^{*}+\frac{E_{s}^{*}-E_{f}^{*}}{1+\left(\frac{t x_{0}}{h_{c}}\right)^{n}} .
$$

As shown in Figure 2, reduced modulus plots for both ITO and HKUST-1 showed a propensity to plateau to a constant $E^{*}$ value as $h_{c} / t$ tended towards zero. For sputter-deposited ITO films, the $1073 \mathrm{~nm}$ film deviated from the general trend toward significantly lower reduced elastic modulus values and was not included in the analysis. This was thought to be due to an extended processing time during magnetron sputtering leading to changes in grain coarsening and defect properties in the ITO films, where such processing relationships for magnetron sputtered films have been described in literature. ${ }^{25,26} \mathrm{X}$-ray diffraction (XRD) measurements confirmed changes in texture with ITO film thickness and some variability in the grain size (see the supplementary material $^{35}$ ). Thicker sputtered ITO films have also been shown to have an increased surface roughness, which may also influence the measurement procedure of the modulus. ${ }^{27}$ Results on thin-films $(<100 \mathrm{~nm})$ were found to be significantly



FIG. 2. Scatter plot of reduced modulus against normalized contact depth for constituent ITO and HKUST-1 thin films with varying thickness, fit with Eq. (1). 
substrate-dependent, and led to reduced modulus values roughly equivalent to that of the substrate, and were also discounted from the analysis. As such, ITO data were modelled using a film thickness range of $122-473 \mathrm{~nm}$. Data fitting of the reduced elastic modulus led to values of $99.1 \pm 11.2 \mathrm{GPa}$, giving an $E$ of $96.7 \pm 10.1 \mathrm{GPa}$ when utilizing a Poisson's ratio of $0.33 .{ }^{27}$ The Hardness for the ITO films was found to be $4.96 \pm 0.50 \mathrm{GPa}$ (the $1073 \mathrm{~nm}$ film proving to be significantly softer, $2.24 \pm 0.26 \mathrm{GPa}$ ). These results for $E$ and $H$ align well with already reported literature values for sputtered ITO thin films. ${ }^{27,28}$

For LPE grown HKUST-1 films, results showed significant substrate influence above $h_{c} / t 0.25$, and when fit with Eq. (1) led to an indentation and elastic modulus of $26.7 \pm 0.6 \mathrm{GPa}$ and $22.0 \pm 0.5 \mathrm{GPa}$, respectively, when taking into account a Poisson's ratio of 0.433 from literature. ${ }^{29}$ Such an elastic modulus value is significantly higher than obtained by Bundschuh et al. (9.3 GPa) for $1 \mu \mathrm{m}$ thick HKUST-1 films grown using the same method, ${ }^{17}$ Tafipolsky et al. $(10.03 \mathrm{GPa})$ for molecular mechanics force-field modelling, ${ }^{29}$ and Van de Voorde et al. $(3.5 \pm 2.5 \mathrm{GPa})$ for electrochemically grown HKUST-1 with a thickness of $11-15 \mu \mathrm{m} .{ }^{18}$ The hardness of the HKUST-1 films was found to be $0.72 \pm 0.17 \mathrm{GPa}$ which is also significantly higher than the previously reported values $(0.23 \pm 0.04 \mathrm{GPa}$ (Ref. 17) and $0.17 \pm 0.16 \mathrm{GPa}$ (Ref. 18)), also perhaps due to the influence of anisotropy and extended processing on film defects for the much thicker HKUST-1 films previously studied. ${ }^{17,18}$ This presents perhaps some evidence for size effects in MOF thin films, while additionally fulfilling the criteria required for coatings in future microelectronic systems $(\geq 10 \mathrm{GPa}){ }^{30}$ The role of size effects on the mechanical properties has been investigated extensively for thin films, where hardening and toughening mechanisms have been observed for thinner films due to dislocation density fields, flow stress effects, and grain or textural effects among other reasons. ${ }^{31}$ Regarding microstructural effects, XRD spectra were measured for HKUST-1 thin films grown on ITO, however, negligible variation was found with film thickness (see the supplementary material ${ }^{35}$ ), however the film had a preferential [111] crystallographic orientation compared to the [100] orientation for Bundschuh et al., ${ }^{17}$ and randomly oriented polycrystalline film of Van de Voorde et al. ${ }^{18}$ Alternatively, the apparent compliance of thicker films could be due to inherent effects of the MOF structure. Several recent publications looking at periodic lattice structures have indicated that repeat lattice layers, analogous to the cubic HKUST-1 MOF structure, can influence mechanical response. ${ }^{32,33}$ Structures containing more repeat units are more compliant due to a decreased influence of the substrate boundary, leading to reduced geometrical hardening from lateral expansion of both nodes and struts. ${ }^{32}$ A reduced compressive strength with more repeat unit layers has also been attributed to a transition from yielding to increased strut buckling. ${ }^{33}$ Such influences on the mechanical response of SURMOF structures are a focus of current investigation.

Multilayered systems were fabricated with the following HKUST-1 and ITO layer thicknesses: blue -5 bilayers of $62 \mathrm{~nm}$ ITO and $80 \mathrm{~nm}$ HKUST-1; green-5 bilayers of $85 \mathrm{~nm}$ ITO and $115 \mathrm{~nm}$ HKUST-1; and yellow-5 bilayers of $100 \mathrm{~nm}$ ITO and $135 \mathrm{~nm}$ HKUST-1. For multilayered devices incorporating alternating layers of these materials, elastic mismatch between layers is an important factor, which can be quantified as $\left(E_{\text {ITO }}^{*}-E_{\text {HKUST-I }}^{*}\right) /\left(E_{\text {ITO }}^{*}+E_{\text {HKUST-I }}^{*}\right)$ assuming that the Young's modulus of the sublayers are identical. Based on the results presented for both ITO and the MOF, we arrive at a mismatch value of 0.58 . Additionally, the volume fraction (relative layer thicknesses) of ITO for the blue, green, and yellow systems is effectively constant $(0.43-0.44)$. It is noted that a multilayer system with a compliant minority phase is always stable, ${ }^{34}$ and in our case as the thinner ITO phase is stiffer stability is then strongly dependent on the interfacial properties.

The multi-layer stacks were then mechanically probed using a Berkovich indenter tip at both small applied forces $(<500 \mu \mathrm{N})$ and larger forces $(10 \mathrm{mN})$. For small forces, a significant decrease was observed in the system elastic modulus (see the supplementary material ${ }^{35}$ ) and hardness (blue $1.39 \pm 0.35 \mathrm{GPa} ;$ green $0.81 \pm 0.33 \mathrm{GPa} ;$ and yellow $0.58 \pm 0.18 \mathrm{GPa}$ ). It should be noted that while such values are not absolute, they give a good indication of the indentation behavior as a function of depth. The observed hardness approaches that of pure HKUST-1 with increasing layer thickness, indicating that much of the strain energy is localized within the more compliant HKUST-1 layers. This observation was verified using elastic simulations of the normal strain and von Mises stress in the layered systems (see the supplementary material ${ }^{35}$ ). The reduced modulus trend also followed the thin-film model of Perriot and Barthel, demonstrating significant stiffening with increasing relative film thickness, again likely due to substrate effects. At forces less than $500 \mu \mathrm{N}$, there was no obvious correlation between contact depth and applied force for the three optical stacks, however, substantial differences in contact depth were observed when applying a $10 \mathrm{mN}$ indentation load. As shown in Figure 3, at $10 \mathrm{mN}$ load blue multi-layered systems had a contact depth of $290 \mathrm{~nm}$, while green multilayered stacks were compressed to $420 \mathrm{~nm}$ and yellow to $580 \mathrm{~nm}$. This equates to $h_{c} / t_{M L}$ values of $0.41,0.42$, and 0.49 , respectively, suggesting that the thicker multi-layer films should be increasingly affected by substrate hardening effects. However this is not observed, as thicker optical stacks tended to be much more compliant. Simulation of the three systems assuming solely elastic deformation under a $10 \mathrm{mN}$ load was also performed, and it was found that there existed no substantial differences in strain localization or contact depth as a result of component layer thickness (Figure 3(a)). Strain was again found to be localized predominantly in the HKUST-1 layers. Differences in the simulated and observed contact depths can be explained by the action of plastic deformation.

In order to better understand the mechanistic differences for deformation of the optical devices, $10 \mathrm{mN}$ indents were observed via topographical scanning using the nanoindenter tip (Figure 3(c)), and in the scanning electron microscope (SEM, Figure 3(d)). From SEM observation, no adhesive failure was observed for any of the photonic sensor systems, along with no evidence of interfacial sliding or delamination. It was found, however, that at large loading thinner multilayered systems "piled-up" to a greater extent than thicker systems; the deformation of the multilayered systems varies 
(ai)
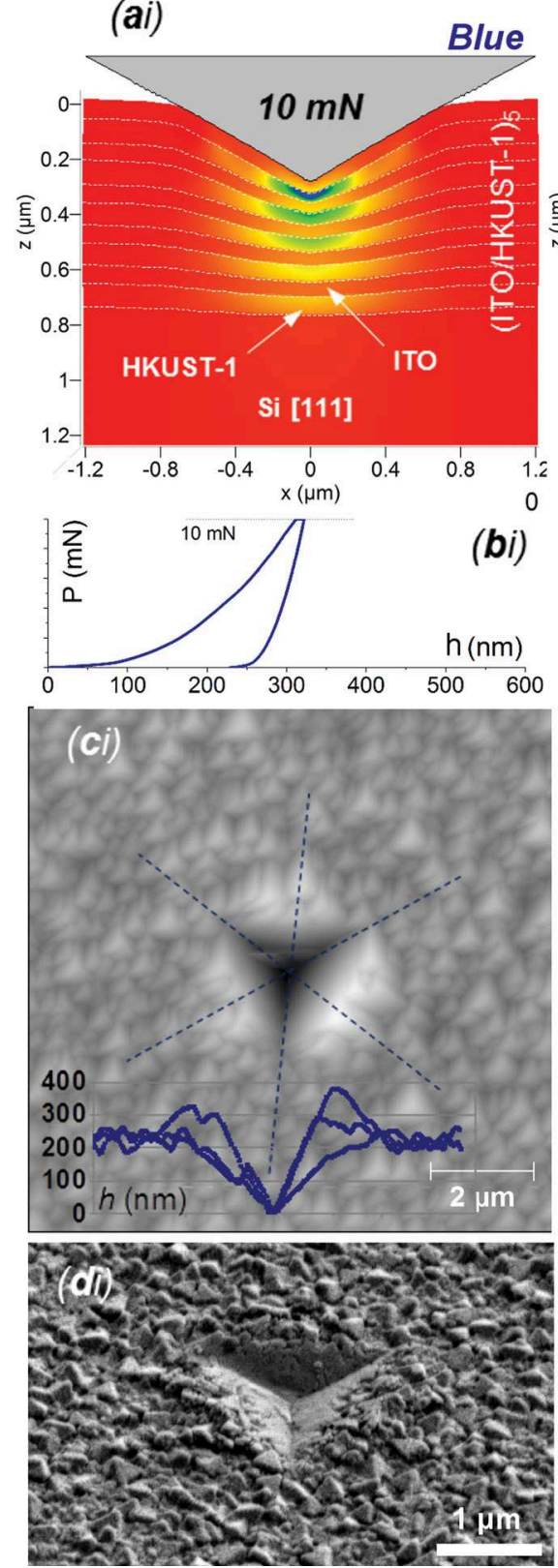

(aii)
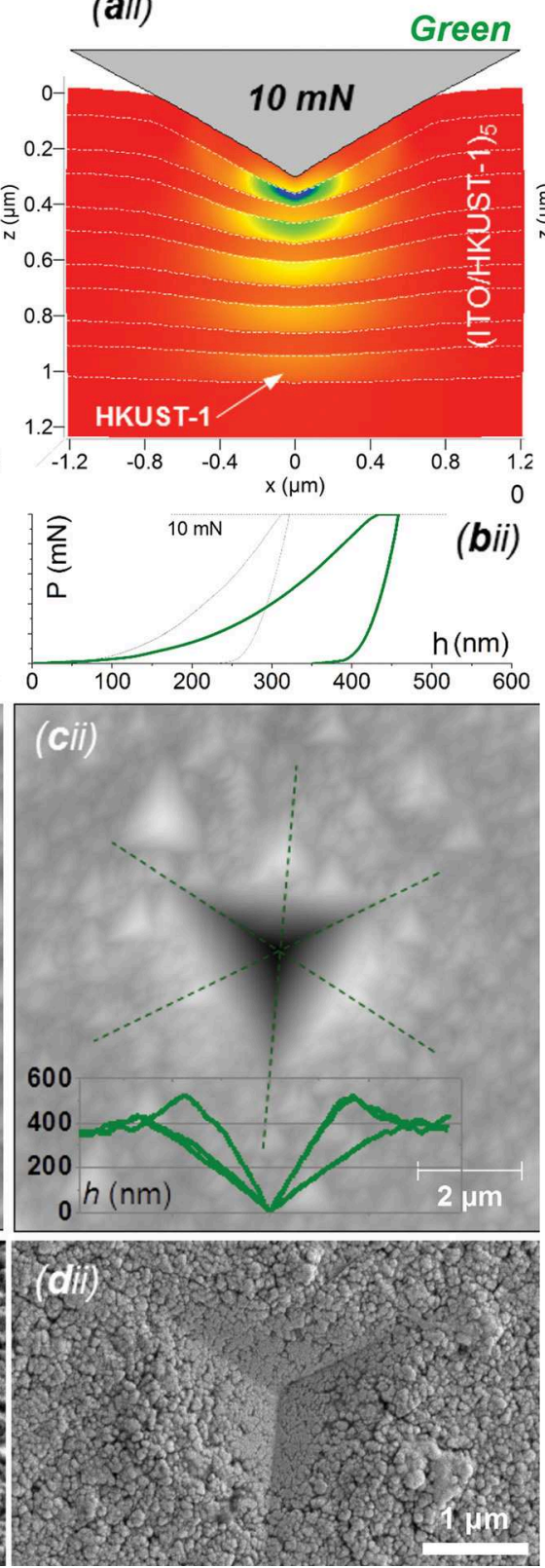

(aiii)
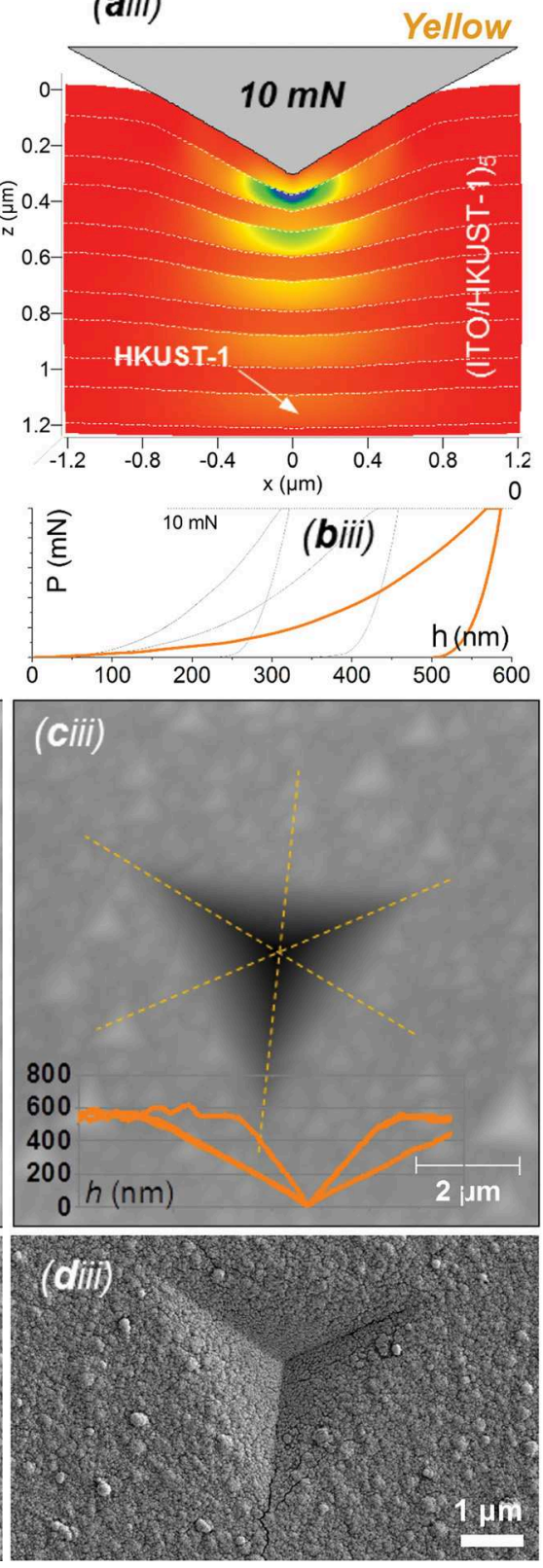

FIG. 3. Hybrid multilayer films under $10 \mathrm{mN}$ Berkovich loading. Elastic simulations (a) of normal strain in the indentation axis for blue (i), green (ii), and yellow (iii) samples (color range -0.61 to 0 strain). Samples were loaded providing force-deformation curves (b), topographical analyses (c), and electron microscopy images (d) of formed residual indents. SEM image (di) was taken at a tilting angle of $45^{\circ}$ to accentuate the observed pile-up.

with the component layer thicknesses. Purely regarding the mechanical properties of the constituent materials, there is no reason to suggest significant differences in deformation behavior, and indeed this is shown in performed elastic simulations where only a slight increase in strain localization in HKUST-1 layers is observed for thinner component layers. Such variations could be due to variable morphology between the three systems; thicker layered systems are observably (from topographical and SEM images) much smoother than thinner systems, indicating that interfacial roughness is likely to change as the layer thickness changes as shown in the cross-sectional SEM image in Figure 1(a) (also see the supplementary material ${ }^{35}$ ). From these images, a greater concentration of discontinuities and structural defects are observed in thinner systems, possibly leading to hardening mechanisms through mechanical interlocking, and pile-up through constrained plasticity which is then not observed for thicker component layers. AFM analysis shows that HKUST-1 thin-films become smoother with increasing film thickness, with the exception of the $190 \mathrm{~nm}$ film which starts become rough again due to the large number of LPE cycles, while XRD has shown textural changes for ITO films with increasing thickness (see the supplementary material ${ }^{35}$ ). Subtle interfacial changes could then influence the presence of defects in the multilayered structure and give rise to a significant change in the elastic-plastic deformation behavior. As previously discussed, the apparent compliance of thicker film systems could also be due to inherent structural effects of the MOF, where much of the strain energy is localized.

In this work, the mechanical properties of single layered thin ITO and HKUST-1 films were studied using nanoindentation, and then the mechanical knowledge gleaned was 
transferred to five-bilayer multilayer systems whereby exploring their "soft/hard" interfaces. Experiments conducted have shown that in multilayered hybrid HKUST-1/ ITO optical devices, the influence of the component material film thickness has a significant effect on the deformation characteristics of the device under uniaxial nanoindentation loading; even for a constant relative film thickness. Such a significant effect dramatically highlights the importance of engineering the interface for thin-film devices. We believe this mechanical analysis of hybrid SURMOF containing multilayered systems will have broad implications for the investigation of other thin-film devices.

J.P.B. would like to acknowledge funding from the EMPA Postdoc program cofounded by FP7: Marie Curie Actions. E.R. thanks the Alexander von Humboldt (AvH) Foundation as well as KIT and CMM for financial support and funding. J.L. and Z.W. thank the China Scholarship Council (CSC) for financial aid. S.W., E.R., and H.G. acknowledge financial support from the Landesstiftung Baden-Württemberg. This work was funded within the priority program SPP 1362 of the German Research Foundation (DFG).

${ }^{1}$ H. Li, M. Eddaoudi, M. O'Keeffe, and O. Yaghi, Nature 402, 276 (1999).

${ }^{2}$ O. Yaghi, G. Li, and H. Li, Nature 378, 703 (1995).

${ }^{3}$ G. Férey and C. Serre, Chem. Soc. Rev. 38, 1380 (2009).

${ }^{4}$ H. K. Arslan, O. Shekhah, J. Wohlgemuth, M. Franzreb, R. A. Fischer, and C. Wöll, Adv. Funct. Mater. 21, 4228 (2011).

${ }^{5}$ O. Shekhah, J. Liu, R. A. Fischer, and C. Wöll, Chem. Soc. Rev. 40, 1081 (2011).

${ }^{6}$ S. Kitagawa, R. Kitaura, and S. Noro, Angew. Chem., Int. Ed. Engl. 43, 2334 (2004).

${ }^{7}$ O. K. Farha, A. Ö. Yazaydın, I. Eryazici, C. D. Malliakas, B. G. Hauser, M. G. Kanatzidis, S. T. Nguyen, R. Q. Snurr, and J. T. Hupp, Nat. Chem. 2, 944 (2010).

${ }^{8}$ J. Liu, W. Zhou, J. Liu, I. Howard, G. Kilibarda, S. Schlabach, D. Coupry, M. Addicoat, S. Yoneda, Y. Tsutsui, T. Sakurai, Z. Wang, P. Lindemann, E. Redel, T. Heine, and C. Wöll, Angew. Chem., Int. Ed. Engl. 54, 7441 (2015).

${ }^{9}$ J.-R. Li, R. J. Kuppler, and H.-C. Zhou, Chem. Soc. Rev. 38, 1477 (2009).
${ }^{10}$ J. Liu, P. K. Thallapally, B. P. McGrail, D. R. Brown, and J. Liu, Chem. Soc. Rev. 41, 2308 (2012).

${ }^{11}$ M. Yoon, R. Srirambalaji, and K. Kim, Chem. Rev. 112, 1196 (2011).

${ }^{12}$ O. Shekhah and M. Eddaoudi, Chem. Commun. 49, 10079 (2013).

${ }^{13}$ S. Eslava, L. Zhang, S. Esconjauregui, J. Yang, K. Vanstreels, M. R. Baklanov, and E. Saiz, Chem. Mater. 25, 27 (2013).

${ }^{14}$ E. Redel, Z. Wang, S. Walheim, J. Liu, H. Gliemann, and C. Wöll, Appl. Phys. Lett. 103, 091903 (2013).

${ }^{15}$ L. Heinke, M. Tu, S. Wannapaiboon, R. A. Fischer, and C. Wöll, Microporous Mesoporous Mater. 216, 200 (2015).

${ }^{16}$ J. C. Tan and A. K. Cheetham, Chem. Soc. Rev. 40, 1059 (2011).

${ }^{17}$ S. Bundschuh, O. Kraft, H. K. Arslan, H. Gliemann, P. G. Weidler, and C. Wöll, Appl. Phys. Lett. 101, 101910 (2012).

${ }^{18}$ B. Van de Voorde, R. Ameloot, I. Stassen, M. Everaert, D. De Vos, and J.C. Tan, J. Mater. Chem. C 1, 7716 (2013).

${ }^{19} \mathrm{Z}$. Li and H. C. Zeng, J. Am. Chem. Soc. 136, 5631 (2014).

${ }^{20}$ S. K. Mishra, D. Verma, S. Bysakh, and L. C. Pathak, J. Nanomater. 2013, 949416 (2013).

${ }^{21}$ J. Liu, E. Redel, S. Walheim, Z. Wang, V. Oberst, J. Liu, S. Heissler, A. Welle, M. Moosmann, T. Scherer, M. Bruns, H. Gliemann, and C. Wöll, Chem. Mater. 27, 1991 (2015).

${ }^{22}$ W. C. Oliver and G. M. Pharr, J. Mater. Res. 7, 1564 (1992).

${ }^{23}$ A. Perriot and E. Barthel, J. Mater. Res. 19, 600 (2004).

${ }^{24}$ W. A. Brantley, J. Appl. Phys. 44, 534 (1973).

${ }^{25}$ N. Arshi, J. Lu, C. G. Lee, J. H. Yoon, B. H. Koo, and F. Ahmed, Bull. Mater. Sci. 36, 807 (2013).

${ }^{26}$ H. N. Shah, V. Chawla, R. Jayaganthan, and D. Kaur, Bull. Mater. Sci. 33, 103 (2010).

${ }^{27}$ T. Wittkowski, J. Jorzick, H. Seitz, B. Schröder, K. Jung, and B. Hillebrands, Thin Solid Films 398, 465 (2001).

${ }^{28}$ K. Zeng, F. Zhu, J. Hu, L. Shen, K. Zhang, and H. Gong, Thin Solid Films 443, 60 (2003).

${ }^{29}$ M. Tafipolsky, S. Amirjalayer, and R. Schmid, J. Phys. Chem. C 114, 14402 (2010).

${ }^{30}$ K. Maex, M. R. Baklanov, D. Shamiryan, F. Lacopi, S. H. Brongersma, and Z. S. Yanovitskaya, J. Appl. Phys. 93, 8793 (2003).

${ }^{31}$ Y. Choi and S. Suresh, Acta Mater. 50, 1881 (2002).

${ }^{32}$ C. I. Hammetter, R. G. Rinaldi, and F. W. Zok, J. Appl. Mech. 80, 041015 (2013).

${ }^{33}$ S. Babaee, B. H. Jahromi, A. Ajdari, H. Nayeb-Hashemi, and A. Vaziri, Acta Mater. 60, 2873 (2012).

${ }^{34}$ N. Sridhar, J. M. Rickman, and D. J. Srolovitz, J. Appl. Phys. 82, 4852 (1997).

${ }^{35}$ See supplementary material at http://dx.doi.org/10.1063/1.4930141 for Fig. S1 and experimental details for film fabrication; Fig. S2 for ITO XRD analysis and AFM analysis in Fig. S5 and Table S2; Fig. S3, Fig. S4, Fig. S5, and Table S1 give information on the properties of HKUST-1 thin films; Fig. S6 shows the evaluation for small forces on multilayer systems; and Fig. S7 shows cross-sectional SEM images of the multilayered systems. 